Vol. 01, No. 02, abril - junio 2021, 22-41

\title{
Evaluación de la estabilidad por pruebas aceleradas, de la cáscara de camu camu (Myrciaria dubia Mc Vaugh) secada por aire caliente
}

\section{Stability evaluation by accelerated tests, of the camu camu husk (Myrciaria dubia Mc Vaugh) dried by hot air}

Jaime E. Basilio-Atencio ${ }^{1, a, *}$, Anthony Paduro-Contreras ${ }^{1, b}$

\author{
${ }^{1}$ Facultad de Ingeniería en Industrias Alimentarias, Universidad Nacional Agraria de la Selva, Carretera Central km. 1.21; \\ Tingo María; Huánuco, Perú. \\ a Ph.D., \jaime.basilio@unas.edu.pe, (D) https://orcid.org/0000-0002-7006-7724 \\ b Ing., $\bigotimes$ gabrielpancon@hotmail.com, (D) https://orcid.org/0000-0001-7112-1772 \\ * Autor de Correspondencia: Tel. +51 968942048 \\ http://dx.doi.org/10.25127/riagrop.20212.676
}

http://revistas.untrm.edu.pe/index.php/RIAGROP revista.riagrop@untrm.edu.pe

Recepción: 22 de enero 2021

Aprobación: 23 de marzo 2021

Este trabajo tiene licencia de Creative Commons. Attribution-NonCommercial-ShareAlike $\quad 4.0$ International Public License - CC-BY-NC-SA 4.0

\section{Resumen}

En el presente estudio se evaluó la estabilidad mediante pruebas aceleradas, de la cáscara de camu camu (Myrciaria dubia Mc Vaugh) secado con aire caliente a $50{ }^{\circ} \mathrm{C}$ con una velocidad de $2.5 \mathrm{~m} / \mathrm{s}$. La cáscara seca del camu camu fue almacenada a 40,50 y $60^{\circ} \mathrm{C}$, durante 45 días y cada 5 días se evaluó el contenido de vitamina $\mathrm{C}$, polifenoles totales por el método de folin-Ciocalteu y la capacidad antioxidante por el método de Brand-Williams. Los datos experimentales obtenidos fueron ajustados a los modelos de cinética de orden de reacción 0,1 y 2 para determinar la constante de velocidad de reacción $(K)$ y a la ecuación de Arrhenius para determinar la energía de activación $(E a)$ del deterioro de los compuestos bioactivos; también se determinó el tiempo de vida media. Los compuestos biactivos de la cáscara seca de camu camu de mejor conservaron durante 45 días a la menor temperatura de almacenamiento $\left(40{ }^{\circ} \mathrm{C}\right)$. La cinética de deterioro de vitamina $\mathrm{C}$, polifenoles totales y capacidad antioxidante de la cáscara de camu camu, mostraron mejores ajustes a un orden de reacción uno, con coeficientes de 
determinación $\left(\mathrm{R}^{2}\right)$ superior a 0.94 . La Ea para la vitamina $\mathrm{C}$, polifenoles totales y capacidad antioxidante variaron entre 8.8466 y $26.5149 \mathrm{~kJ} / \mathrm{mol}$ y fue menor para polifenoles totales y mayor para la capacidad antioxidante. La vida útil de todos los compuestos bioactivos evaluados se redujo con el incremento de la temperatura de almacenamiento.

Palabras clave: Capacidad antioxidante, polifenoles, vida útil, vitamina C.

\begin{abstract}
In the present study, the stability was evaluated by accelerated tests, of the husk of camu camu (Myrciaria dubia Mc Vaugh) dried with hot air at $50{ }^{\circ} \mathrm{C}$ with a speed of $2.5 \mathrm{~m} / \mathrm{s}$. The dried camu camu peel was stored at 40, 50 and $60^{\circ} \mathrm{C}$ for 45 days and every 5 days the content of vitamin $\mathrm{C}$, total polyphenols were evaluated by the folinCiocalteu method and antioxidant capacity by the Brand - Williams method. The experimental data obtained were adjusted to the kinetic models of reaction order 0,1 , and 2 to determine the reaction rate constant (K) and to the Arrhenius equation to determine the activation energy (Ae) of the deterioration of the bioactive compounds; the half-life time was also determined. The bioactive compounds of the dried camu camu husk were best preserved for 45 days at the lowest storage temperature $\left(40^{\circ} \mathrm{C}\right)$. The kinetics of deterioration of vitamin C, total polyphenols, and antioxidant capacity of camu camu husk, showed the best adjustments to a reaction order one, with a determination of (R2) higher than 0.94 . The Ae for vitamin C, total polyphenols, and antioxidant capacity varied between 8.8466 and $26.5149 \mathrm{~kJ} / \mathrm{mol}$ and was lower for total polyphenols and higher for antioxidant capacity. The shelf life of all bioactive compounds evaluated was reduced with the increase of the storage temperature.
\end{abstract}

Keywords: Antioxidant capacity, polyphenols, shelf life, vitamin C.

\section{INTRODUCCIÓN}

El camu camu (Myrciaria dubia Mc Vaugh) es un arbusto frutal que se encuentra en las orillas de los ríos y lagos de la Amazonía. Por presentar buenas características agronómicas, tecnológicas y nutracéuticas, el camu camu tiene un gran potencial de mercado, principalmente, debido al alto contenido de ácido ascórbico y otros ingredientes activos que se encuentran en sus frutos y tomaron gran importancia en los mercados como Japón, Francia, Alemania y Estados Unidos (DosSantos et al., 2018)

El camu camu es una fuente potencial de vitamina $C$, que se concentra principalmente en la cáscara del fruto en estado de maduración: maduro y sobremaduro. Actualmente, el camu camu se presenta como una especie promisoria por la alta productividad por área, por la posibilidad de su cultivo en zonas intervenidas, con la ventaja de mejorar la producción en zonas ya colonizadas, y por la demanda creciente en los mercados mundiales (DosSantos et al., 2018).

El camu camu es una fruta que crece en la Amazonía peruana, principalmente en zonas inundables. Su árbol alcanza en promedio 5 metros de altura. La fruta tiene forma globosa y esférica de 1 a $3 \mathrm{~cm}$ de diámetro y $20 \mathrm{~g}$ de peso, aproximadamente. En estado maduro, desarrolla un color café-rojizo a violeta negruzco y una pulpa suave. Alojada en la pulpa, se encuentran tres semillas reniformes de 8 a $5 \mathrm{~mm}$ de largo y 5.5 a $11 \mathrm{~mm}$ de ancho. La pulpa del fruto maduro es comestible, de 
agradable sabor ácido, parecido a la cereza y el limón (Arellano et al., 2016).

El camu camu es una buena fuente de minerales como sodio, potasio, calcio, zinc, magnesio, manganeso, cobre y varias clases de aminoácidos, tales como serina, valina $\mathrm{y}$ leucina. Además, contiene una pequeña cantidad de pectina y almidón. La glucosa y la fructosa son sus azúcares principales del camu camu (Garay y Villafuerte, 2015). Cada $100 \mathrm{~g}$ de cáscara de camu camu contiene: proteínas $0.4 \mathrm{~g}$; carbohidratos $5.9 \mathrm{~g}$; fécula $0.44 \mathrm{~g}$; vitamina $\mathrm{C}$ $2145 \mathrm{mg}$, agua $84.1 \mathrm{~g}$; azúcar $1.28 \mathrm{~g}$; fibra alimenticia $1.1 \mathrm{~g}$; calcio $15.7 \mathrm{mg}$; cobre $0.2 \mathrm{mg}$; hierro $0.53 \mathrm{mg}$; magnesio $12.4 \mathrm{mg}$; manganeso $2.1 \mathrm{mg}$; grasa $0.2 \mathrm{~g}$; potasio $83.8 \mathrm{mg}$; sodio 11.1 mg y zinc 0.36 mg (Paucar, 2012).

Los componentes bioactivos son ingredientes funcionales de los alimentos, capaces de aportar efectos beneficiosos a la salud, influyen en la actividad celular, en los mecanismos fisiológicos y reducen el riesgo a enfermedades crónicas. Por lo tanto, la presencia de diferentes compuestos bioactivos en la cáscara de camu camu podría ser utilizado para retardar o prevenir diversas enfermedades cardiovasculares y el cáncer (Valencia y Guevara, 2013).

Dentro de los principales compuestos bioactivos que se encuentran en la cáscara del camu camu son la vitamina $\mathrm{C}$ de aproximadamente $3000 \mathrm{mg} / 100 \mathrm{~g}$, que superan a la naranja (92 mg/100g de pulpa) y limón (44.2 $\mathrm{mg} / 100 \mathrm{~g}$ de pulpa) y polifenoles (Chang, 2013; Monteiro, 2014; Muñoz et al., 2015), compuestos que se deterioran fácilmente con el incremento de la temperatura y la oxidación (Badui, 2013).

Una de las alternativas para proteger los compuestos activos y prevenir el deterioro de los productos hortofrutícolas es el secado, que existen varios tipos, como el secado por conducción directa e indirecta, convectivo por aire caliente, por radiación electromagnética (microonda), transferencia de calor (Valcarcel, 2014). Dentro de estos tipos, destaca el secado convectivo por aire caliente, donde el calor se suministra a través de aire caliente, el cual fluye sobre la superficie del sólido (Bautista y Valdivieso, 2016).

Para evitar el deterioro de los productos hortofrutícolas y conservarlos por largo tiempo, se someten a uno de los procesos, o a una combinación de ellos, basados en altas temperaturas (escaldado, pasteurización, esterilización), en bajas temperaturas (refrigeración, congelación), en la eliminación del agua (deshidratación), en el control de la actividad del agua (concentración, alimentos de humedad intermedia), en el empleo de aditivos (benzoatos, sorbatos), en el control de $\mathrm{pH}$ (acidificación, fermentación), en el uso de radiaciones, entre otras (Badui, 2013).

En los casos en donde la calidad del alimento decrece lentamente bajo las condiciones reales de almacenamiento, resulta conveniente realizar estudios de vida útil mediante el método acelerado. Este método consiste en almacenar el alimento bajo condiciones ambientales que aceleren el deterioro (como, por ejemplo, temperatura o humedad mayores) y luego interpolar o extrapolar la vida útil a las condiciones usualmente experimentales por el producto (Cuastumal et al., 2016).

De la literatura revisada, se evidencia que no existe información del deterioro de las propiedades bioactivas de la cáscara de camu camu, por tanto, el objetivo del presente estudio fue evaluar la estabilidad por pruebas 
aceleradas de secado la cáscara de camu camu por aire caliente.

\section{MATERIALES Y MÉTODOS}

\subsection{Lugar de ejecución}

El presente trabajo se llevó a cabo en la Universidad Nacional Agraria de la Selva (UNAS). Los análisis se realizaron en el laboratorio de Análisis de alimentos, laboratorio Ingeniería de alimentos y el Centro de Investigación para el Desarrollo Biotecnológico de la Amazonía (CIDBAM).

\subsection{Materia prima y reactivos}

Se usó camu camu (Myrciaria dubia Mc Vaugh) en estado maduro proveniente de la ciudad de Pucallpa, distrito de Yarinacocha, provincia de Coronel Portillo, departamento de Ucayali, caserío San Juan, ubicado a orillas del río Yarinacocha cuya ubicación geográfica está definida por las coordenadas de $8^{\circ} 19^{\prime} 19.89^{\prime \prime}$ latitud Sur y a $74^{\circ} 36^{\prime} 7.61^{\prime \prime}$ latitud Oeste, se cosechó durante los meses de diciembre a febrero.

Los reactivos que se emplearon fueron: Ácido oxálico $\left(\mathrm{C}_{2} \mathrm{H}_{2} \mathrm{O}_{4} 2 \mathrm{H}_{2} \mathrm{O}\right)$ pureza $99.5 \%$; ácido ascórbico $\quad\left(\mathrm{C}_{6} \mathrm{H}_{8} \mathrm{O}_{6}\right) \quad$ pureza $99.5 \%$; 2,4 diclorofenol-indofenol, folin \& Ciocalteu's phenol reagent, HI-LR 1,9-2,1 N; carbonato de sodio $\left(\mathrm{Na}_{2} \mathrm{CO}_{3}\right)$ pureza $99.5 \%$; DPPH $1 \mathrm{mM}(2,2-$ diphenyl-1-picrylhydrazyl); ácido gálico $\left(\mathrm{C}_{7} \mathrm{H}_{6} \mathrm{O}_{5} \mathrm{H}_{2} \mathrm{O}\right)$ pureza 99.5\%; 6-Hydroxy-2,5,7,8tetramethyl-chromane-2-carboxylic acid; todos adquiridos de la marca Merk.

\subsection{Métodos de análisis}

2.3.1. Cuantificación de ácido ascórbico: método espectrofotométrico descrito por Monteiro (2014).
2.3.2. Cuantificación de polifenoles totales: método reportado por Caisahuana (2012).

2.3.3. Cuantificación de antioxidantes: método descrito por Hung y Yen (2012).

\subsubsection{Determinación del orden de reacción (n) y} constante de velocidad de reacción $(k)$ : por el modelo de integración de pérdida de calidad planteado por Gonzales et al. (2016).

\subsection{Metodología experimental}

\subsubsection{Obtención de la cáscara de camu camu}

En la Figura 1, se muestran las operaciones para la obtención de cáscara de camu camu seca.

En esta investigación, se utilizaron $7 \mathrm{~kg}$ de camu camu. Los frutos con residuos extraños y con daño mecánico fueron separados, luego seleccionados los de mejor apariencia. Luego se lavaron las frutas en una bandeja con $10 \mathrm{~L}$ de agua para eliminar las partículas extrañas. Seguidamente, las frutas se pelaron de forma manual para la obtención de la cáscara y pulpa, la cáscara fue puesta en mallas metálicas de 20 x $25 \mathrm{~cm}$ de área para ser secadas en un secador de bandejas por aire caliente, a $50{ }^{\circ} \mathrm{C}, 2.5 \mathrm{~m} / \mathrm{s}$ de velocidad de aire por aproximadamente 6 horas hasta peso constante (Garay y Villanueva, 2015). La cáscara seca de camu camu se trituró en una licuadora, se tamizó en un tamiz \#12 con $1.68 \mathrm{~mm}$ de diámetro de la partícula. Las muestras molidas fueron enfriadas a temperatura ambiente y se empacó en bolsas de polipropileno de baja densidad con espesor 0,03 $\mathrm{mm}$. 


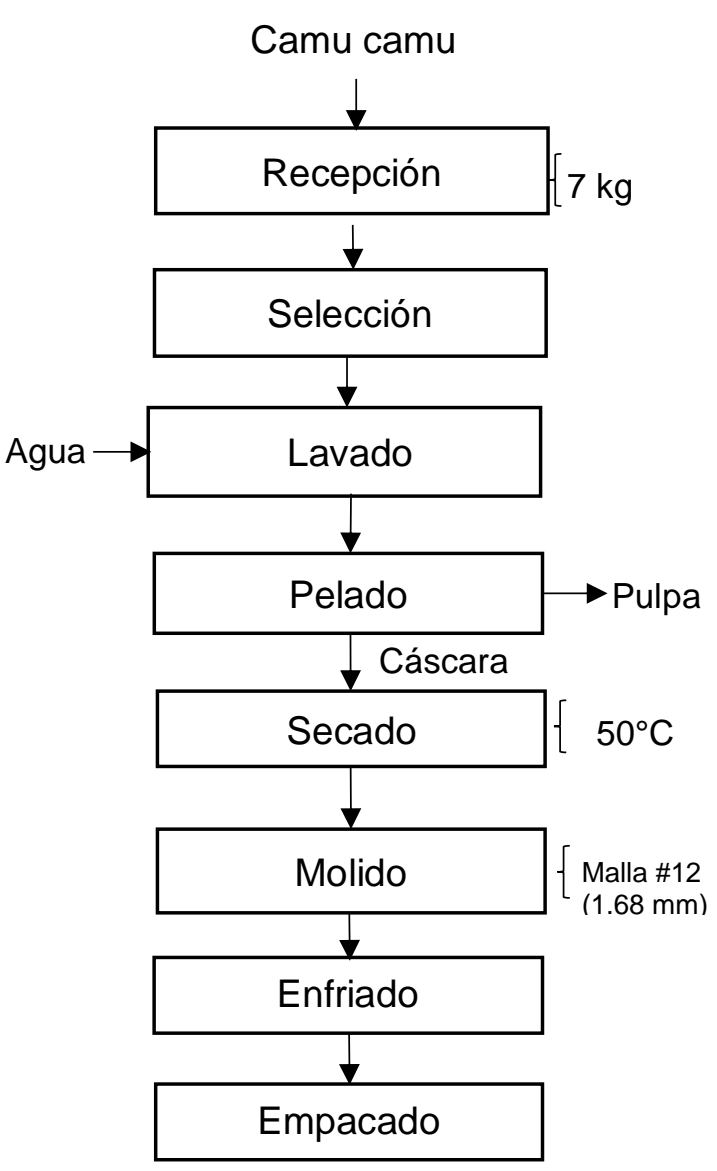

Figura 1. Diagrama de la obtención de la cáscara de camu camu.

2.4.2. Obtención del extracto para análisis de polifenoles totales y capacidad antioxidante

Se pesó $0.5 \pm 0.05 \mathrm{~g}$ de cáscara de camu camu, se llevó a un tubo y se enrazó a $20 \mathrm{~mL}$ con solución etanol/agua $(20 / 10 \mathrm{v} / \mathrm{v})$ y se sometió a agitación constante por 24 horas en ausencia de luz. Se filtró con papel filtro N.ํㅜ 40, la fracción líquida se sometió a centrifugación (10000 rpm/10 minutos a $4{ }^{\circ} \mathrm{C}$ ), se tomó $5 \mathrm{~mL}$ del sobrenadante (extracto) con la cual se procedió a realizar los análisis.

\subsubsection{Evaluaciones durante el almacenamiento}

La cáscara de camu camu en empaques de 5 gramos fueron puesto en estufa a temperaturas de 40,50 y $60{ }^{\circ} \mathrm{C}$, con una variación promedio de $\pm 2{ }^{\circ} \mathrm{C}$. Se evaluó el contenido de vitamina $\mathrm{C}$, polifenoles totales y capacidad antioxidante. El tiempo de almacenamiento, así como los intervalos de tiempo para cada análisis fueron determinados en 5 días para todos los análisis que se realizaron por triplicado y para cada temperatura de trabajo, los resultados se expresaron como valores promedios con su respectiva desviación estándar.

a) Evaluación de Vitamina C: Se elaboró la curva estándar de ácido ascórbico con el colorante 2,6 diclorofenolindofenol, a una concentración de 0,048 $\mathrm{g} / \mathrm{L}$ con agua destilada $1000 \mathrm{~mL}$ y como agente reductor se empleó ácido ascórbico (A.A.) al 0,1\% con una solución de ácido oxálico 0,4\%. Las concentraciones para la curva estándar estuvieron comprendidas entre 10 a $50 \mathrm{mg}$ A.A./100 mL. Después de realizar regresión lineal, se obtuvo un coeficiente de correlación $\left(\mathrm{R}^{2}\right)$ de 0.99 .

Para el análisis de vitamina $C$ se pesó $0.3 \mathrm{~g}$ de cáscara de camu camu. En un mortero, se agregó $10 \mathrm{~mL}$ de ácido oxálico al $0.4 \%$ y se mezcló la muestra hasta que se disolvió por completo. Seguidamente, se colocó en un tubo de ensayo por 30 minutos para que sedimente bajo sombra y se tomó $5 \mathrm{~mL}$ del sobrenadante para ponerlo en tubos de Eppendorf de $1.5 \mathrm{~mL}$. Se centrifugó a 10000 rpm por 10 minutos a $4^{\circ} \mathrm{C}$ y se toma $1 \mathrm{~mL}$ del sobrenadante para el análisis. Se preparó la solución de la muestra diluida 1:40 (tomando como muestra $40 \mu \mathrm{L}+1560 \mu \mathrm{L}$ ácido oxálico al $0.4 \%$ obteniendo un volumen de $1.600 \mu \mathrm{L}$ ). Se colocó en el espectrofotómetro a una longitud de onda de $515 \mathrm{~nm}$ en una cubeta conteniendo $1000 \mathrm{~mL}$ de agua destilada y en la otra $100 \mu \mathrm{L}$ de ácido oxálico al $0.4 \%+900 \mu \mathrm{l}$ de colorante siendo nuestra (L1). Igualmente se hace para (L2) 
con la adición de $100 \mu \mathrm{L}$ la muestra diluida. Todas las lecturas se hicieron por triplicado. El contenido de vitamina $C$ se calculó utilizando la ecuación (01)

$$
A_{515 \mathrm{~nm}}=A_{\text {control }}(L 1)-A_{\text {muestra }}(L 2)
$$

Donde: A es la absorbancia de vitamina C, L1 es la bsorbancia del colorante o control y L2 es la absorbancia de la muestra. El valor obtenido se remplaza en la ecuación dada por la curva estándar. Esta concentración se multiplica por el factor de dilución $1 / 40$ y se obtuvo la concentración de la vitamina $C$ en $\mathrm{mg} / \mathrm{mL}$.

b) Polifenoles totales: Se preparó una solución stock de $5 \mathrm{~mL}$ de ácido gálico a una concentración de $1 \mathrm{mg} / \mathrm{mL}$, a partir de la cual se prepararon concentraciones de: $10,25,50$, 75 y 100 ug/mL. Luego se tomó $500 \mu \mathrm{L}$ de reactivo de Folin Ciocalteu y $400 \mu \mathrm{L}$ de carbonato de sodio $\left(\mathrm{Na}_{2} \mathrm{CO}_{3} 5 \mathrm{H}_{2} \mathrm{O}\right) 7.5 \%$, para cada tratamiento. Se dejó en la oscuridad por $2 \mathrm{~h}$ a temperatura ambiente $\mathrm{y}$ se procedió a leer en el espectrofotómetro UV/VIS a una longitud de onda de $740 \mathrm{~nm}$. Con los resultados, se preparó la curva estándar que obtuvo un $\mathrm{R}^{2}$ de 0.99 . Se tomó $100 \mu \mathrm{L}$ del extracto $(20 / 10 \mathrm{v} / \mathrm{v})$ con el procedimiento similar a la curva estándar. Todas las lecturas se hicieron por triplicado. Los resultados se expresaron como equivalente de ácido gálico (mg EAG/100 g de muestra).

c) Capacidad antioxidante: La curva estándar de Trolox fue preparada a una concentración de 2 mM (0.05 g de Trolox en $100 \mathrm{~mL}$ de etanol al $96 \%$ ), a partir del cual se prepararon las concentraciones ( 0.25 a $1.5 \mathrm{mM})$. Se tomó 25 $\mu \mathrm{L}$ de etanol al $96 \%+975 \mu \mathrm{L}$ de estándar (1, 2,3 y 4) en una cubeta y en otra cubeta (blanco) etanol al $96 \%$, al medir la absorbancia en el espectrofotómetro a 515 $\mathrm{nm}$ tomando el valor a los 10 minutos. Con los resultados obtenidos se graficó la concentración vs absorbancia y se obtuvo un $\mathrm{R}^{2}$ de 0.99 .

Para la determinación de la capacidad antioxidante de las muestras, se tomó $5 \mathrm{~mL}$ de solución stock de DPPH a $1 \mathrm{mM}(0,0394 \mathrm{~g}$ de DPPH en $100 \mathrm{~mL}$ de etanol al 96\%) y se enrazó a $50 \mathrm{~mL}$ de etanol, luego se vertió en frasco ámbar y conservó a $4^{\circ} \mathrm{C}$ protegido de la luz. Por otro lado, se tomó $25 \mu \mathrm{L}$ del extracto de la muestra $+975 \mu \mathrm{L}$ solución de DPPH a $0.1 \mathrm{mM}$, y se midió la absorbancia en el espectrofotómetro para tomar el valor a los 10 minutos o hasta que se observe un valor de absorbancia constante. Todos los análisis se hicieron por triplicado. El valor de absorbancia sirvió para reemplazar y calcular en la ecuación de la curva estándar. Los resultados se expresaron en capacidad antioxidante equivalente a Trolox $(\mu \mathrm{M}$ TEAC /g).

\subsection{Determinación de los parámetros cinéticos}

2.5.1. Orden de reacción (n) y constante de velocidad de reacción

Para determinar el orden de reacción, se emplearon los resultados obtenidos de los contenidos de vitamina C, polifenoles totales y capacidad antioxidante. Se eligió un orden de reacción (n) igual a cero, primer orden $\mathrm{y}$ segundo orden, para luego integrar con la ecuación (02)

$\pm \frac{d A}{d T}=k A^{n}$ 
Donde para $n=0$ se tiene $A=A_{0}+k t$; para $n=1$ se tiene $\ln A=\ln A_{0}+k t$ y para $n=2$ se tiene $\frac{1}{A}=\frac{1}{A_{0}}+k t$.

En las ecuaciones, se reemplazó los resultados promedio experimentales de A (vitamina C, polifenoles totales y capacidad antioxidante), $t$ que es el tiempo de almacenamiento a las temperaturas evaluadas $\left(40,50\right.$ y $\left.60^{\circ} \mathrm{C}\right), k$ es la constante de velocidad de reacción (valor de la pendiente de dicha regresión) y $n$ es el orden de reacción. Se regresionó linealmente para escoger la ecuación ( $n=0, n=1$ y $n=2)$ que mejor se ajusta a los datos experimentales, en base al coeficiente de correlación $\left(\mathrm{R}^{2}\right)$. Se determinó el orden de reacción para cada factor de calidad medido. También se determinaron los valores de las constantes de velocidad de reacción $(k)$ que es igual al valor de la pendiente de dicha regresión para cada temperatura de estudio

\subsubsection{Energía de activación (Ea) mediante el modelo Arrhenius}

Para la evaluación de la energía de activación se usó la ecuación de Arrhenius relacionando la constante de velocidad de reacción $(k)$ con la temperatura (Ecuación 03).

$K=K_{0} e^{\frac{E a}{R T}}$

Al aplicar logaritmo a ambos lados de la ecuación toma una forma lineal, donde al graficar Lnk contra 1/T daría una línea recta cuya pendiente sería igual a $(-E a / R)$, la que nos servirá para determinar la vida útil del alimento (Ecuación 04).

$\operatorname{Ln} K=\operatorname{Ln} K_{0}-\frac{E a}{R(T)}$

Los valores de las constantes de velocidad de reacción obtenidos experimentalmente a sus respectivas temperaturas en grados kelvin $\left({ }^{\circ} \mathrm{K}\right)$, se ajustó al modelo de Arrhenius por regresión.
Luego en la pendiente de la ecuación obtenida se remplazó el valor de $\mathrm{R}=1.98717 \mathrm{cal} \cdot \mathrm{mor}^{-1} \mathrm{~K}^{-1}$, y se despejó la energía de activación Ea (Ecuación 05).

$b=-\frac{E a}{R}$

Donde $b$ es la pendiente de la ecuación y $E a$ es la energía de activación.

\section{RESULTADOS Y DISCUSIÓN}

\subsection{De las evaluaciones en la cáscara de camu camu durante el almacenamiento.}

\subsubsection{Vitamina $C$}

Para la curva estándar de la vitamina C, se usó ácido ascórbico, según Fracassetti et al. (2013). El valor $\mathrm{R}^{2}=0.9912$ obtenido indica un buen ajuste, el cual fue superior al $\mathrm{R}^{2}$ de 0.983 reportado por Klinar et al. (2009).

El contenido de vitamina C durante el almacenamiento a diferentes temperaturas se muestran en la figura 2. Al día cero, la cáscara de camu camu presentó $1825.74 \mu \mathrm{g}$ AA/100 g de camu camu, contenido menor a lo reportado por Arrellano et al. (2016), quienes reportan $2780 \mathrm{mg} / 100 \mathrm{~g}$ y Sotero et al. (2009) quien encontró, en el banco de Germoplasma INIALoreto, $10506.37 \mathrm{mg} / 100 \mathrm{~g}$.

A los 5 y 10 días de almacenamiento, se observó un efecto en el deterioro de la vitamina $\mathrm{C}$ en el almacenamiento a $60{ }^{\circ} \mathrm{C}$. Arellano et al. (2016) sostienen que el ácido ascórbico, es termosensible $\mathrm{y}$ en procesos que implican condiciones de calor puede causar disminución en su contenido. Asimismo, Villareal et al. (2013) indican que el efecto se atribuye a que la vitamina $C$ de la cáscara puede degradarse fácilmente por exposición a la temperatura y por oxidación. 
A los 20 días de almacenamiento a $60{ }^{\circ} \mathrm{C}$, fue donde más se perdió (50.94\% de vitamina C remanente), almacenadas a 50 y $40 \quad{ }^{\circ} \mathrm{C}$ conservaron mejor la vitamina C (73.49 y 75.64 $\%$ de vitamina $C$ remanente, respectivamente). Cuastumal et al. (2016) mencionan que todos los tratamientos de temperatura $(30,40,50,60$ y 70 $\left.{ }^{\circ} \mathrm{C}\right)$, lograron reducir significativamente la vitamina C. Mendoza-Corvis et al. (2017) reportan que el ácido dehidroascórbico se caracteriza por ser uno de los constituyentes más termosensibles en los alimentos y es por eso su disminución en el almacenamiento.
A días 45 de almacenamiento, se observó un descenso considerable de vitamina $\mathrm{C}$, donde la mayor pérdida de vitamina $\mathrm{C}$ es a $60{ }^{\circ} \mathrm{C}$ con $36.08 \%$ de vitamina $\mathrm{C}$ remanente y a $40,50{ }^{\circ} \mathrm{C}$ son los que mejor se conservaron con $56.31 \mathrm{y}$ $50.94 \%$ de vitamina C remanente, respectivamente. De acuerdo con Castañeda et al. (2010), las pérdidas en vitamina C de la cáscara de camu camu pueden aducirse a que el ácido ascórbico es un componente muy sensible a la temperatura (calor) y oxidación.

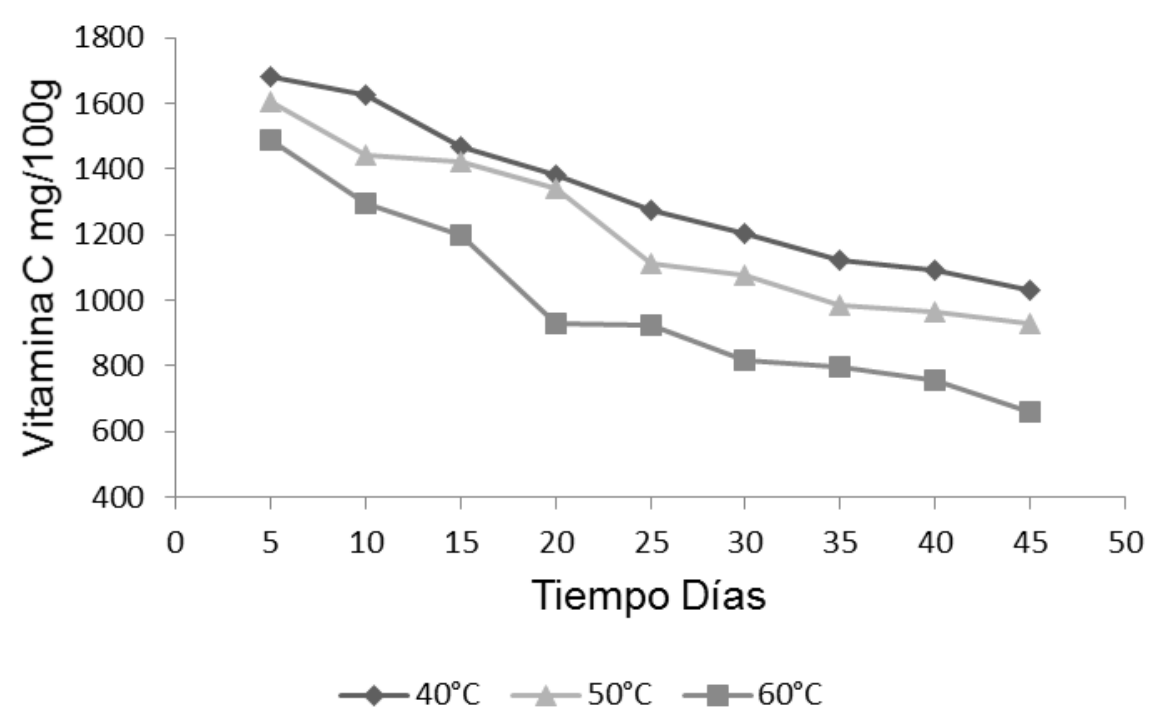

Figura 2. Variación del contenido de vitamina $\mathrm{C}$ a diferentes temperaturas de almacenamiento, durante 45 días.

\subsubsection{Polifenoles totales}

La curva estándar realizada para la cuantificación de polifenoles totales presentó un $\mathrm{R}^{2}=0.9992$, que evidencia un buen ajuste, similar a los reportado por Cabrera (2015) quien encontró un $\mathrm{R}^{2}=0.9996$ mediante el uso de ácido gálico para la curva estándar de los polifenoles. Los datos obtenidos de la evaluación de polifenoles totales durante el almacenamiento a diferentes temperaturas se muestran en la figura 3. 


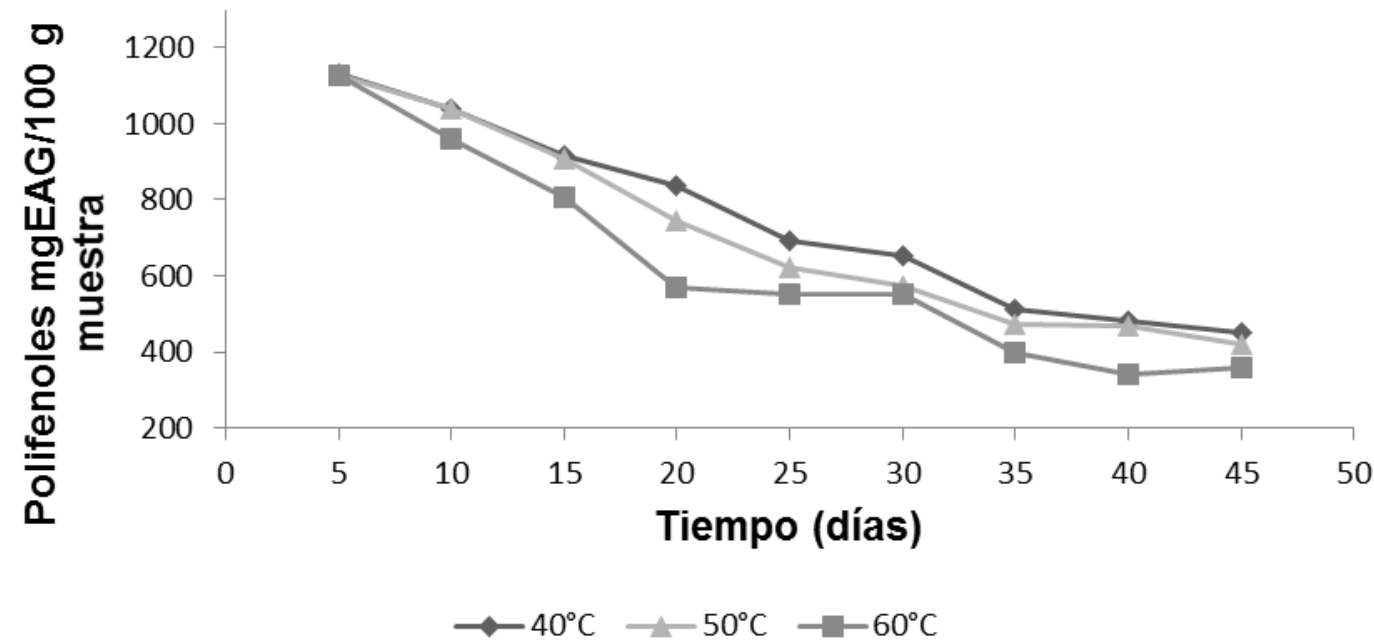

Figura 3. Variación del contenido de polifenoles totales a diferentes temperaturas de almacenamiento, durante 45 días.

En el día cero, se obtuvo 1604.30 mg EAG/100 g de muestra. Ramírez et al. (2012), encontraron el mayor contenido de polifenoles totales (con el ácido gálico) en el microencapsulamiento.

A los 15 días de almacenamiento, a $60{ }^{\circ} \mathrm{C}$, la cáscara seca de camu camu conservó menos los polifenoles obteniendose $50.24 \%$ de polifenoles remanente. En cambio, a 50 y $40{ }^{\circ} \mathrm{C}$ de almacenamiento presentaron mejor conservacion (56.64 y $57.23 \%$ de polifenoles remanente, respectivamente). Según Soto y Barraza (2014), esta fruta cuando es sometida a altas temperaturas sufre pérdida de peso, deterioro significativo y reducción de su vida útil por efecto del acelerado proceso de maduración, que desmejora su apariencia y calidad.

A los 20 días de almacenamiento, a $60{ }^{\circ} \mathrm{C}$, se observó un mayor descenso de polifenoles (35.57 \% de polifenoles remanente), con respecto a 40 y $50{ }^{\circ} \mathrm{C}(52.18$ y $46.34 \%$ de polifenoles remanente, respectivamente). Según Muñoz et al. (2015), se reportó que al incrementar la temperatura puede contribuir a la activación de enzimas que participan en la descomposición de los compuestos complejos que crean puentes de hidrógeno con los compuestos fenólicos.

A los 45 días de almacenamiento, se diferenciaron los porcentajes de polifenoles y se observó que a $60{ }^{\circ} \mathrm{C}$ de almacenamiento, perdió mayor cantidad $(22.32 \%$ de polifenoles remanente), con respecto a los demás tratamientos. Según Cofre (2015), en el tratamiento de 45 y 55 minutos, la concentración disminuyó considerablemente, debido a la inestabilidad de los polifenoles totales a prolongados tiempos de exposición a altas temperaturas.

\subsubsection{Capacidad antioxidante (DPPH)}

La curva estandar del reactivo DPPH con las diferentes concentraciones trolox como patron tuvo un buen ajuste $\left(R^{2}=0.9987\right)$. El $R^{2}$ encontrado fue similar a lo encontrado por Cedeño (2017), quien reportó un $\mathrm{R}^{2}=0.9905$, quien usó el reactivo de Trolox a concentraciones de 250, 500, 750 y $1000 \mu \mathrm{mol}$ Trolox/ $\mu \mathrm{L}$. 
La capacidad antioxidante de la cascara seca de camu camu evaluada durante el almacenamiento a diferentes temperaturas se muestran en la figura 4.
(2015), quienes realizaron estudios similares para DPPH y detectaron la máxima actividad antioxidante de camu-camu $\quad(5848.90 \mu \mathrm{M}$ TEAC/g de muestra de cáscara).

En el día cero, se obtuvo $2208.92 \mu \mathrm{M}$ TEAC/g valor inferior al mencionado por Neves et al.

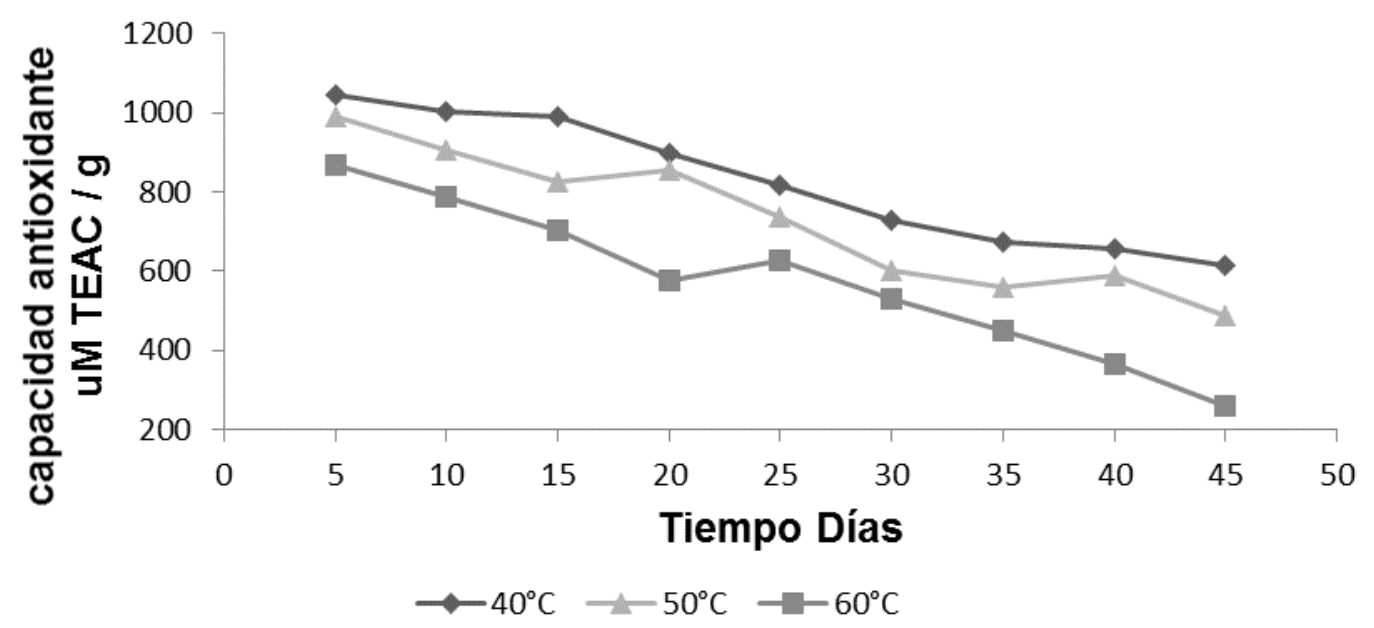

Figura 4. Variación de la capacidad antioxidante a diferentes temperaturas de almacenamiento, durante 45 días.

A los 5 días, se encontró diferencias en el almacenamiento a $60{ }^{\circ} \mathrm{C}$ con una capacidad antioxidante de $39.26 \%$ respecto al inicial, que fue inferior a los otras temperatura de 40 y 50 ${ }^{\circ} \mathrm{C}$ con 47.37 y $44.7 \%$, respectivamente y se constató que la temperatura es un factor muy influyente en la capacidad antioxidante. Según Estrada et al. (2014), estas pérdidas pueden deberse a que durante la deshidratación por secado y se establece una transferencia de masa de dos vías: el agua y algunas sustancias naturales solubles.

Al los 25 días, se observó que a $60^{\circ} \mathrm{C}$ hay una tendencia a descender la capacidad antioxidante con respeto a los $40{ }^{\circ} \mathrm{C}$ que tienden a mantenerse. Zapata et al. (2015) determinaron que la pérdida de la actividad antioxidante era del $4 \%$ en secados por miniencapsulamiento.
A los 45 días de almacenamiento se observó que hay variación entre las temperaturas de $40^{\circ} \mathrm{C}$ con una capacidad antioxidante de $615.39 \mu \mathrm{M}$ TEAC/g, y a $60{ }^{\circ} \mathrm{C}$ con $258.54 \mu \mathrm{M}$ TEAC/g con una pérdida considerable de la capacidad antioxidante. Busos (2016) observó que cuando la capacidad antioxidante es sometida a bajas temperatura y a un tiempo de almacenamiento existe una relación directa en el descenso de los polifenoles.

\subsection{Parámetros cinéticos en el almacenamiento}
3.2.1. Orden de reacción (n) y constante de velocidad de reacción ( $k$ ) para el deterioro de la vitamina $\mathrm{C}$

El orden de reacción en un alimento se puede calcular en función a la concentración de los reactantes o de los productos. Se evalúa la velocidad o rapidez de las reacciones de 
deterioro, expresados como cambios de concentración por unidad de tiempo.

Para cada grupo de datos de concentraciones de vitamina $C$, se elaboraron gráficos que incluyeron la concentración de vitamina C frente a tiempo (orden 0 ) tal como se muestra en la figura 2. Otra figura de logaritmo natural de concentración de vitamina $C$ frente a tiempo en orden 1 como se puede observar en las figuras $5 \mathrm{~A}$. Finalmente un gráfico de $1 /$ concentración de vitamina $C$ frente a tiempo en orden 2 como se muestra en la figura 5B. El criterio para determinar el orden de reacción fue el que más se ajustó al modelo, cuantificado por el coeficiente de correlación $\left(\mathrm{R}^{2}\right)$. Gonzales et al. (2016) indicaron que el olor extraño, brillo, sabor característico, consistencia y jugosidad del camu camu, se ajustaron mejor a cinética de primer orden, mientras que el resto de atributos siguieron una cinética de orden cero.

Los valores de la constante de velocidad de reacción $K$ de la concentración de vitamina $C$ en el almacenamiento ajustado a los modelos de la cinética de orden cero, primer orden y segundo orden, se muestra en la tabla 1.

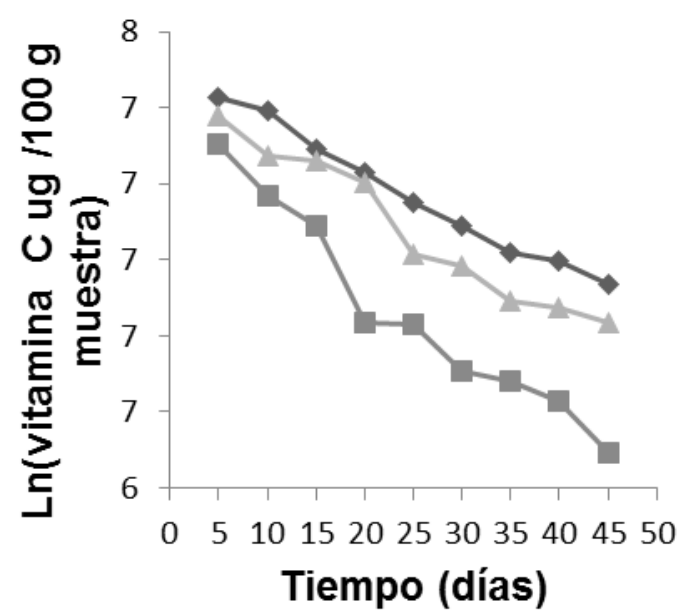

$\neg-40^{\circ} \mathrm{C}-50^{\circ} \mathrm{C}-60^{\circ} \mathrm{C}$

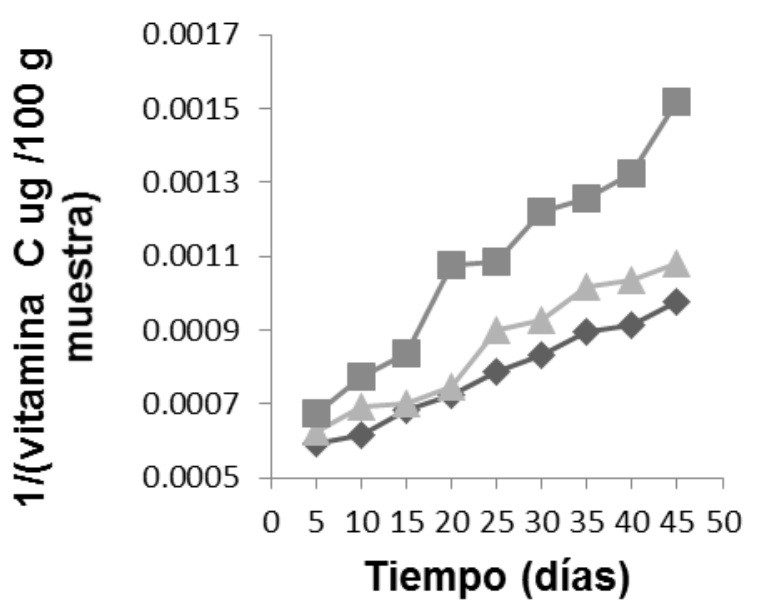

$\because 40^{\circ} \mathrm{C} \longrightarrow 50^{\circ} \mathrm{C}-60^{\circ} \mathrm{C}$

(B)

Figura 5. Ajuste de los valores experimentales de degradación de vitamina $\mathrm{C}$ a los modelos de cinética orden 1(A) y orden 2(B).

Tabla 1. Constante de velocidad de reacción $(K)$ de la concentración de vitamina $C$ de la cáscara de camu camu en el almacenamiento

\begin{tabular}{ccccccc}
\hline \multirow{2}{*}{$\mathbf{T}^{\mathbf{0}} \mathbf{C}$} & \multicolumn{2}{c}{ Orden 0 } & \multicolumn{2}{c}{ Orden 1 } & \multicolumn{2}{c}{ Orden 2 } \\
\cline { 2 - 7 } & $\mathbf{K}$ & $\mathbf{R}^{\mathbf{2}}$ & $\mathbf{K}$ & $\mathbf{R}^{\mathbf{2}}$ & $\mathbf{K}$ & $\mathbf{R}^{\mathbf{2}}$ \\
\cline { 2 - 7 } $\mathbf{4 0}$ & -16.926 & 0.974 & -0.013 & 0.988 & 0.000010 & 0.994 \\
$\mathbf{5 0}$ & -17.563 & 0.947 & -0.014 & 0.959 & 0.000012 & 0.966 \\
$\mathbf{6 0}$ & -19.491 & 0.914 & -0.019 & 0.953 & 0.000020 & 0.971 \\
\hline
\end{tabular}


De acuerdo al factor de correlación $\left(\mathrm{R}^{2}\right)$, los datos experimentales se ajustan mejor a un modelo de cinética de primer orden con un valor de $\mathrm{R}^{2}$ promedio de 0.967 , similar a lo reportado por Gonzales et al. (2016) quienes obtuvieron en cinética de orden 1 con $\mathrm{R}^{2}$ de 0.988 .

Energía de activación para la degradación de la vitamina $C$

Las constantes de velocidad de reacción $(K)$ de orden cero, uno y dos fueron acondicionadas para el ajuste del modelo de Arrhenius que se muestra en la tabla 2.

Con los valores de regresión al modelo de Arrhenius, se determinó la energía de activación ( $E a)$. Los parámetros de Arrhenius para la degradación de vitamina $C$ en la cáscara de camu camu se muestra en la tabla 3. Adicionalmente, en la figura 6, se muestra la constante de velocidad de reacción $(K)$ de orden uno para la concentración de vitamina $C$ ajustada a la ecuación de Arrhenius.

Los valores de constante de velocidad de degradación de vitamina $C(K)$ que mejor se ajustaron al modelo de Arrhenius fueron el de orden uno con $\mathrm{R}^{2}$ de 0.9408 (tabla 2). Según Terry (2015), el deterioro de vitamina C a $20 \mathrm{y}$ $50{ }^{\circ} \mathrm{C}$ presentó un factor de correlación de 0.9128 y 0.9723 y se aplicó un análisis de regresión exponencial primer orden.

Finalmente, el modelo matemático que representó el comportamiento del deterioro de vitamina $\mathrm{C}$, a la temperatura de $20^{\circ} \mathrm{C}$ fue:

$\ln$ vitamina $C=\ln$ vitamina $C_{\text {inicial }}-0.0078 t_{\text {días }}$

Tabla 2. Constante de velocidad de reacción $(K)$ de vitamina $C$ en el almacenamiento

\begin{tabular}{cccccc}
\hline & Temperatura & \multicolumn{3}{c}{ Constante de velocidad de reacción $\mathbf{k}$} \\
\hline $\mathbf{T}{ }^{\mathbf{0}} \mathbf{C}$ & ${ }^{\mathbf{0}} \mathbf{K}$ & $\mathbf{1 /}{ }^{\circ} \mathbf{K}$ & Orden $\mathbf{0}$ & Orden $\mathbf{1}$ & Orden $\mathbf{2}$ \\
\hline $\mathbf{4 0}$ & 313 & 0.0032 & 16.926 & 0.0128 & 0.000010 \\
$\mathbf{5 0}$ & 323 & 0.0031 & 17.563 & 0.0145 & 0.000012 \\
$\mathbf{6 0}$ & 333 & 0.0030 & 19.491 & 0.0194 & 0.000020 \\
\hline \hline
\end{tabular}

Tabla 3. Parámetros de Arrhenius para la degradación de vitamina C en la cáscara de camu camu

\begin{tabular}{cccc}
\hline Orden de reacción $(\mathbf{n})$ & $\mathbf{K}_{\mathbf{0}}$ & $\mathbf{E a}(\mathbf{K J} / \mathbf{m o l})$ & $\mathbf{R}^{\mathbf{2}}$ \\
\hline Orden cero & 173.24 & 6.08289 & 0.9203 \\
Orden uno & 12.233 & 17.9343765 & 0.9408 \\
Orden dos & 1.3053 & 30.830166 & 0.9419 \\
\hline \hline
\end{tabular}




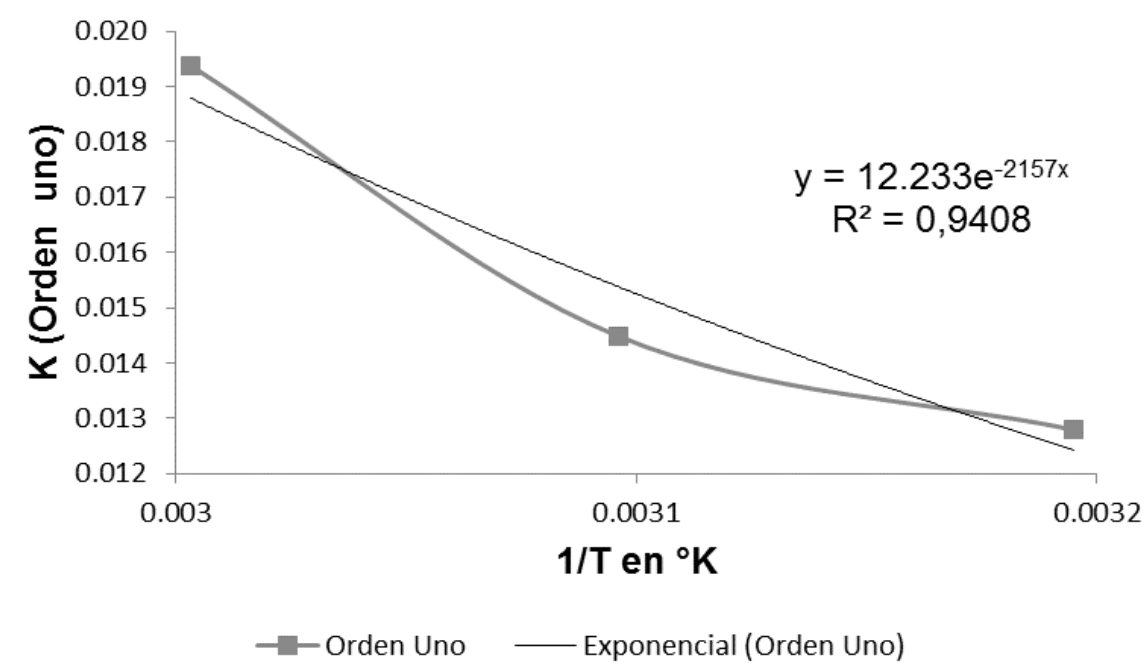

Figura 6. Constante de velocidad de reacción $(\mathrm{K})$ orden uno para la concentración de vitamina C ajustada a la ecuación de Arrhenius.

3.2.2. Orden de reacción (n) y constante de velocidad de reacción $(k)$ para el deterioro de polifenoles totales.

Para cada grupo de datos de concentración de polifenoles totales, se elaboraron gráficos, concentración de polifenoles totales vs tiempo en orden 0 , (figura 3), logaritmo natural de concentración de polifenoles totales vs tiempo en orden 1 (figura 7A). Finalmente, $1 /$ concentración de polifenoles totales vs tiempo en orden 2 como se muestra en la figura 7B. El criterio para determinar el orden de reacción fue el que más se ajustó al modelo, cuantificado por el coeficiente de correlación $\left(R^{2}\right)$.

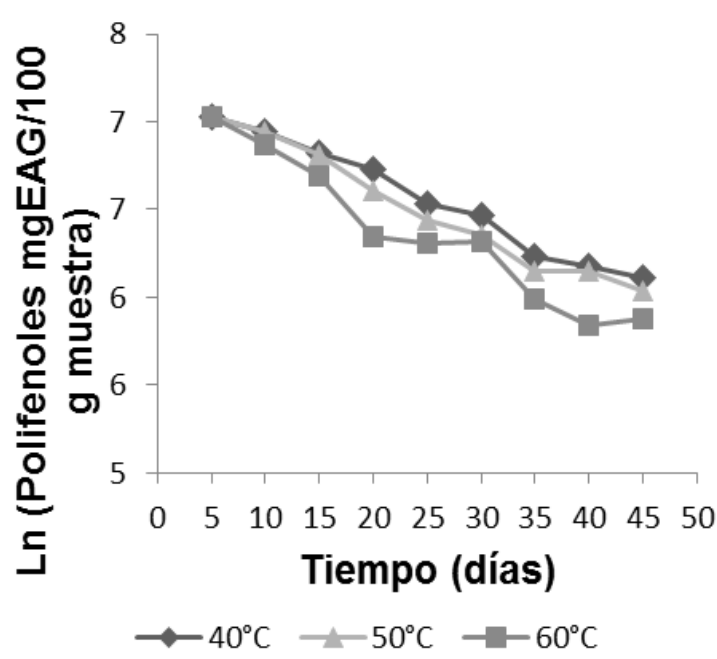

(A)

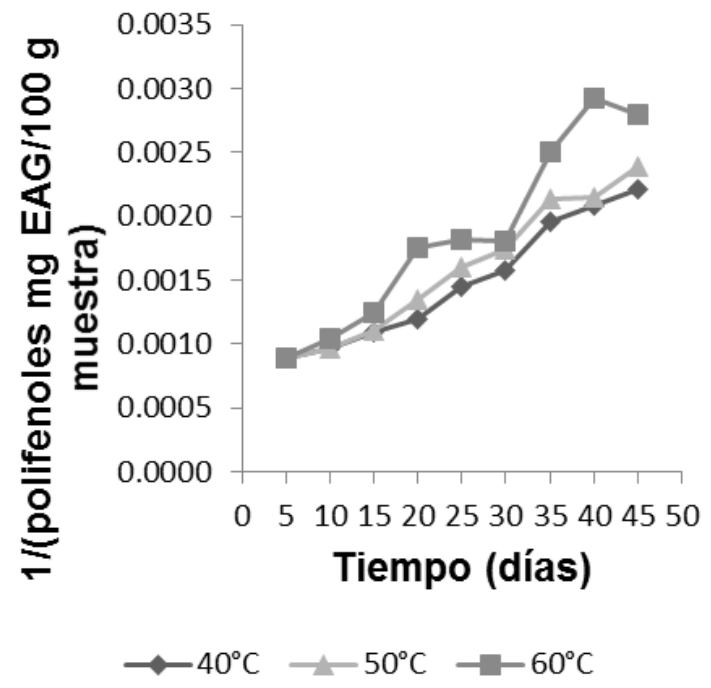

(B)

Figura 7. Ajuste de los valores experimentales de degradación de polifenoles totales a los modelos de cinética Orden 1 (A) y orden 2 (B). 
Los valores de la concentración de polifenoles totales fueron ajustados a los modelos de la cinética de orden cero, uno y dos, el resultado de estos ajustes se muestra en tabla 4 . Torres y Vidaurre (2015) obtuvieron como resultado de polifenoles una cinética de orden uno, dando una energía de activación de $13.4571 \mathrm{Kcal} / \mathrm{mol}$. De acuerdo a los resultados obtenidos, el deterioro de la polifenoles totales corresponde a la cinética de orden uno.
Energía de activación para la degradación de los polifenoles

La constante de velocidad de reacción $(K)$ a orden cero, primero y segundo a diferentes temperaturas acondicionadas para el ajuste del modelo de Arrhenius, se muestra en la tabla 5. Por otro lado, en la tabla 6, se presenta los parámetros de Arrhenius para la degradación de polifenoles totales en la cáscara de camu camu. En la figura 8, se muestra la constante de velocidad de reacción $(K)$ de concentración de polifenoles totales en ecuación de orden uno, ajustada a la ecuación de Arrhenius.

Tabla 4. Constante de velocidad de reacción $(K)$ de la concentración de polifenoles totales de la cáscara de сати сати

\begin{tabular}{ccccccc}
\hline \multirow{2}{*}{$\mathbf{T}^{\mathbf{o}} \mathbf{C}$} & \multicolumn{2}{c}{ Orden 0 } & \multicolumn{2}{c}{ Orden 1 } & \multicolumn{2}{c}{ Orden 2 } \\
\cline { 2 - 7 } & $\mathbf{K}$ & $\mathbf{R}^{2}$ & $\mathbf{K}$ & $\mathbf{R}^{\mathbf{2}}$ & $\mathbf{K}$ & $\mathbf{R}^{\mathbf{2}}$ \\
\cline { 2 - 7 } $\mathbf{4 0}$ & -17.974 & 0.976 & -0.0247 & 0.9858 & 0.000036 & 0.973 \\
$\mathbf{5 0}$ & -18.676 & 0.949 & -0.0264 & 0.9794 & 0.000040 & 0.982 \\
$\mathbf{6 0}$ & -19.184 & 0.903 & -0.0304 & 0.9497 & 0.000053 & 0.945 \\
\hline \hline
\end{tabular}

Tabla 5. Constante de velocidad de reacción $(K)$ de polifenoles totales para orden 0,1 y 2

\begin{tabular}{cccccc}
\hline \multicolumn{3}{c}{ Temperatura } & \multicolumn{4}{c}{ Constante de velocidad de reacción $\mathbf{k}$} \\
\hline $\mathbf{T}^{\mathbf{0}} \mathbf{C}$ & ${ }^{\mathbf{0}} \mathbf{K}$ & $\mathbf{1 /}{ }^{\mathbf{0}} \mathbf{K}$ & Orden $\mathbf{0}$ & Orden $\mathbf{1}$ & Orden $\mathbf{2}$ \\
\hline $\mathbf{4 0}$ & 313 & 0.0032 & 17.974 & 0.0247 & -0.000036 \\
$\mathbf{5 0}$ & 323 & 0.0031 & 18.676 & 0.0264 & -0.000040 \\
$\mathbf{6 0}$ & 333 & 0.0030 & 19.184 & 0.0304 & -0.000053 \\
\hline \hline
\end{tabular}

Tabla 6. Parámetros de Arrhenius para la degradación de polifenoles totales en la cáscara de camu camu

\begin{tabular}{cccc}
\hline Orden de reacción $(\mathbf{n})$ & $\mathbf{K}_{\mathbf{0}}$ & $\mathbf{E a}(\mathrm{KJ} / \mathbf{m o l})$ & $\mathbf{R}^{2}$ \\
\hline Orden cero & 53.33 & 2.82609855 & 0.993 \\
Orden uno & 0.7323 & 8.846628 & 0.9493 \\
Orden dos & 0.0873 & 0.00000025 & 0.9003 \\
\hline \hline
\end{tabular}




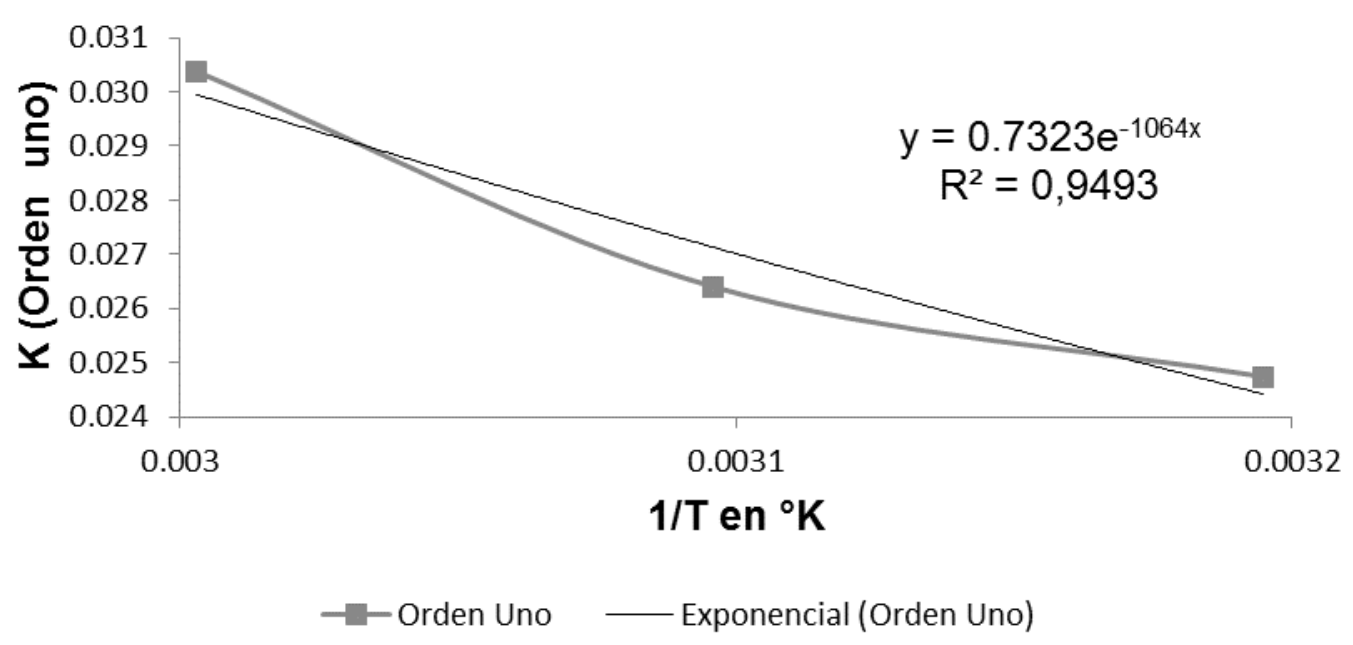

Figura 8. Constante de velocidad de reacción $(\mathrm{K})$ de concentración de polifenoles totales en ecuación de orden uno, ajustada a la ecuación de Arrhenius.

De la tabla 6, se puede afirmar que el valor de $K$, que mejor se ajusta al modelo de Arrhenius, es el de orden uno. Valdez-Hernandez et al. (2015) obtuvieron una energía de activación de $11,47 \mathrm{~kJ} / \mathrm{mol}$ en cinética de orden uno para los polifenoles totales.

\subsubsection{Orden de reacción (n) y constante de velocidad} de reacción $(k)$ para el deterioro de la capacidad antioxidante.

Se elaboraron gráficos de concentración de capacidad antioxidante vs tiempo en orden 0 (figura 4), logaritmo natural de concentración de capacidad antioxidante vs tiempo en orden 1 (figura 9A), 1/concentración de capacidad antioxidante vs tiempo en orden 2, (Figura 9B). El criterio para determinar el orden de reacción, que más se ajustó al modelo, fue por el coeficiente de correlación $\left(\mathrm{R}^{2}\right)$.

El resultado del ajuste se muestra en la tabla 7. Cofre (2015) obtuvo un coeficiente de determinación $\mathrm{R}^{2}=0.72$ a un nivel de confianza de $95 \%$, mientras que el valor de $\mathrm{R}^{2}$ obtenido fue de 0.9357 .

Energía de activación para la degradación de los antioxidantes

La constante de velocidad de reacción $(\mathrm{K})$ a orden cero, uno y dos acondicionadas para el ajuste del modelo de Arrhenius se muestra en la tabla 8.

El ajuste de los valores de velocidad de reacción (K) de diferentes órdenes de reacciones al modelo de Arrhenius y la energía de activación se muestra en tabla 9. Según Sanchez et al. (2015), los datos obtenidos se ajustan a la velocidad de reacción de orden uno, con un $K$ igual a 0.0675 para $90^{\circ} \mathrm{C}$ de almacenamiento. En la figura 10, se muestra la constante de velocidad de reacción $(K)$ de concentración de la capacidad antioxidante en orden de reacción uno, ajustada a la ecuación de Arrhenius. 


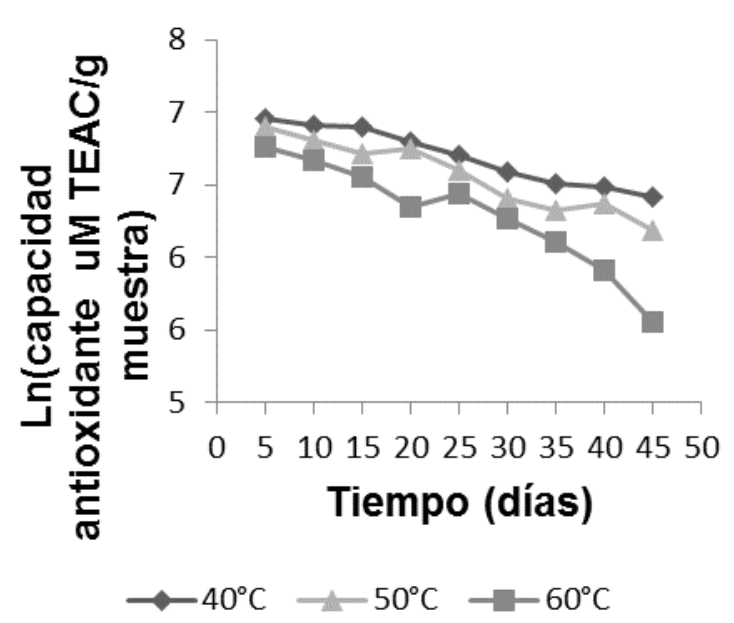

(A)

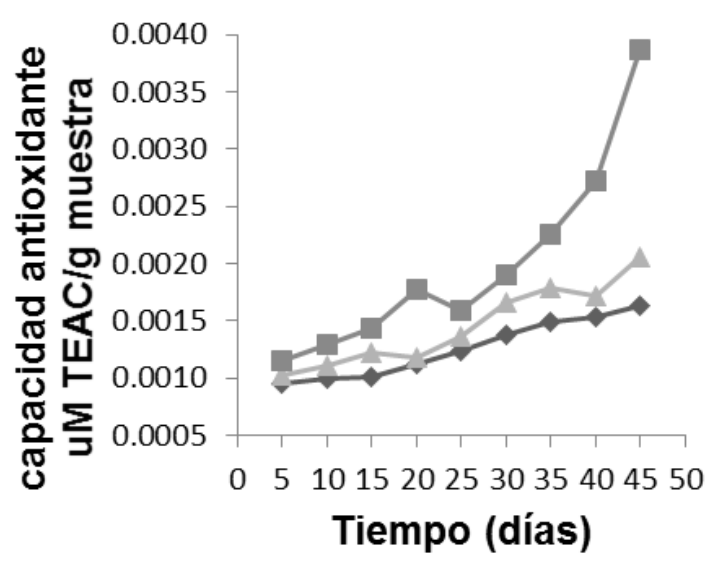

$-40^{\circ} \mathrm{C}-50^{\circ} \mathrm{C}-60^{\circ} \mathrm{C}$

(B)

Figura 9. Ajuste de los valores experimentales de capacidad de antioxidantesa los modelos de cinetica Orden 1 (A) y 2 (B).

Tabla 7. Constante de velocidad de reacción $(K)$ de la concentración de capacidad antioxidante de la cáscara de camu camu

\begin{tabular}{ccccccc}
\hline \multirow{2}{*}{$\mathbf{T}^{\mathbf{o}} \mathbf{C}$} & \multicolumn{2}{c}{ Orden 0 } & \multicolumn{2}{c}{ Orden 1 } & \multicolumn{2}{c}{ Orden 2 } \\
\cline { 2 - 7 } & $\mathbf{K}$ & $\mathbf{R}^{\mathbf{2}}$ & $\mathbf{K}$ & $\mathbf{R}^{2}$ & $\mathbf{K}$ & $\mathbf{R}^{\mathbf{2}}$ \\
\cline { 2 - 7 } $\mathbf{4 0}$ & -11.902 & 0.972 & -0.015 & 0.975 & 0.000018 & 0.973 \\
$\mathbf{5 0}$ & -12.449 & 0.946 & -0.017 & 0.944 & 0.000025 & 0.929 \\
$\mathbf{6 0}$ & -14.210 & 0.969 & -0.027 & 0.923 & 0.000057 & 0.817 \\
\hline \hline
\end{tabular}

Tabla 8. Constante de velocidad de reacción $(K)$ de la capacidad antioxidante en el almacenamiento

\begin{tabular}{cccccc}
\hline & Temperatura & \multicolumn{3}{c}{ Constante de velocidad de reacción $\boldsymbol{k}$} \\
\hline $\mathbf{T}^{\mathbf{0}} \mathbf{C}$ & ${ }^{\circ} \mathbf{K}$ & $\mathbf{1} /{ }^{\circ} \mathbf{K}$ & Orden $\mathbf{0}$ & Orden $\mathbf{1}$ & Orden $\mathbf{2}$ \\
\hline $\mathbf{4 0}$ & 313 & 0.0032 & 11.902 & 0.0146 & -0.000018 \\
$\mathbf{5 0}$ & 323 & 0.0031 & 12.449 & 0.0175 & -0.000025 \\
$\mathbf{6 0}$ & 333 & 0.0030 & 14.210 & 0.0270 & -0.000057 \\
\hline \hline
\end{tabular}

Tabla 9. Parámetros de Arrhenius para la degradación de la capacidad antioxidante en la cáscara de camu

\begin{tabular}{cccc}
\multicolumn{4}{l}{ сатu } \\
\hline Orden de reacción $(\mathbf{n})$ & $\mathbf{K}_{\mathbf{0}}$ & $\mathbf{E a}(\mathbf{K J} / \mathbf{m o l})$ & $\mathbf{R}^{2}$ \\
\hline Orden cero & 0.0000009 & 0.023729583 & 0.9204 \\
Orden uno & 371.79 & 26.5149405 & 0.9357 \\
Orden dos & 0.1981 & 0.00000049 & 0.8696 \\
\hline \hline
\end{tabular}




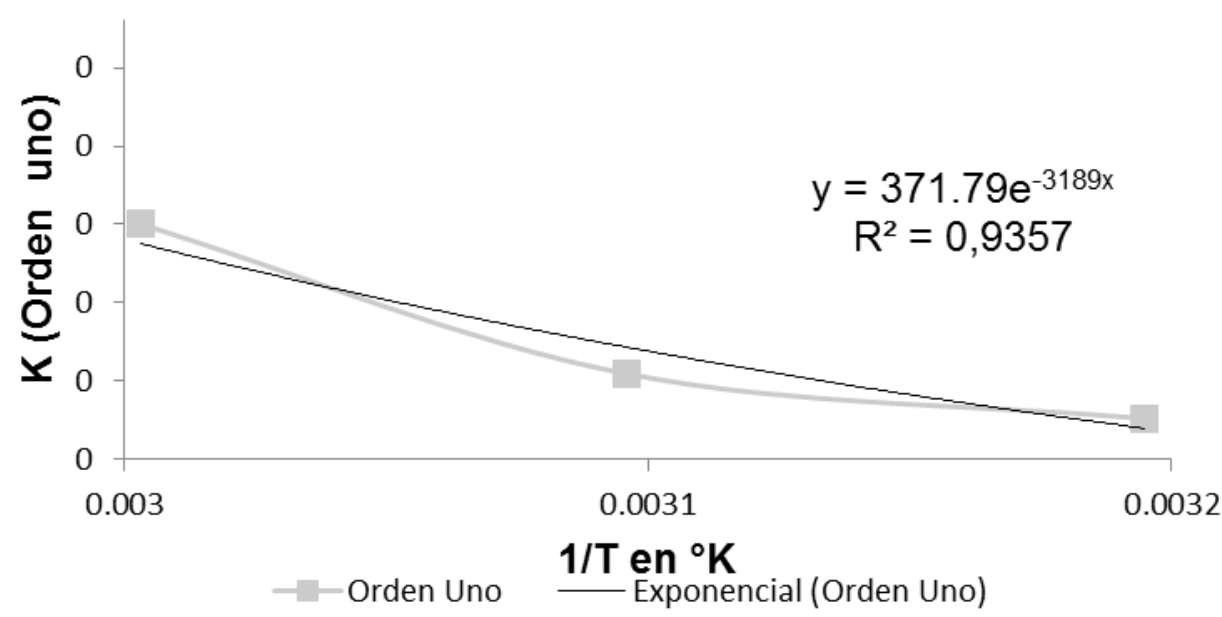

Figura 10. Constante de velocidad de reacción $(\mathrm{K})$ de concetración de la capacidad antioxidante en orden de reacción uno, ajustada a la ecuación de Arrhenius.

De la tabla 9, se puede afirmar que los valores de $K$, que mejor se ajustaron al modelo de Arrhenius, fueron de orden uno.

Según Cofre (2015), los cambios en la calidad de los alimentos son reportados en la literatura mediante modelos cinéticos de degradación de orden cero y de orden uno.

\subsection{Vida útil de la cáscara de camu camu}

Se define el tiempo de vida media como el tiempo necesario para que reaccione la mitad de su concentración inicial. Suele representarse como $t_{1 / 2}$ (ecuación 06).

$t_{1 / 2} \gg A=\frac{A_{0}}{2}$

Para una reacción de orden cero, se calcula mediante la ecuación integrada (07) y para una reacción de primer orden (ecuación 08), donde $t$ es el tiempo y $k$ es la velocidad de reacción.

$$
\begin{aligned}
& t_{1 / 2}=\frac{A_{0}}{2 k} \\
& t_{1 / 2}=\frac{\ln 2}{k}
\end{aligned}
$$

La vida útil media de un alimento es el periodo de tiempo durante el cual se mantiene una calidad adecuada siempre que se garanticen las condiciones de conservación. De esta manera, se hace para vitamina $C$, polifenoles totales $y$ capacidad antioxidante.

Se observa la descomposición química no enzimática por oxidación de la vitamina $C$, con el uso de la ecuación integrada de primer orden (ecuación 08) y el reemplazo de los valores $K$ de la tabla 1 , se obtuvo el tiempo de vida media para vitamina $C$, que se muestran en la tabla 10. Del mismo modo, se calculó para polifenoles totales y capacidad antioxidante, con el uso de los valores $k$ de las tablas 4 y 7 , respectivamente, cuyos valores del tiempo de vida media se muestran en la tabla 10. 
Tabla 10. Tiempo de vida media de la cáscara de camu camu, medidos según su contenido de vitamina $C$, polifenoles totales y capacidad atioxidante, a diferentes temperaturas

\begin{tabular}{cccc}
\hline & \multicolumn{3}{c}{ Tiempo de vida media (días) } \\
\cline { 2 - 4 } Temperatura $\left({ }^{\circ} \mathbf{C}\right)$ & Vitamina C & $\begin{array}{c}\text { Polifenoles } \\
\text { totales }\end{array}$ & $\begin{array}{c}\text { Capacidad } \\
\text { antioxidante }\end{array}$ \\
\hline 40 & 54 & 28 & 47 \\
50 & 47 & 26 & 39 \\
60 & 35 & 22 & 25 \\
\hline
\end{tabular}

El análisis de la vitamina $\mathrm{C}$ es uno de los más utilizados como indicador de la calidad nutricional, ya que esta es altamente vulnerable a la oxidación química, enzimática y muy soluble en agua, por lo que es un indicador sensible y apropiado para evaluar los cambios en la calidad durante el transporte, procesado y almacenaje de hortalizas y frutas. Según Badui (2013), la vitamina C es la más termolábil e inestable, usada como índice de retención de nutrientes, es decir que, si a resiste a los tratamientos térmicos durante el procesamiento de alimentos, todos los demás nutrimentos serán poco afectados.

Por su lado, los polifenoles pueden ser afectados por la temperatura a que son sometidos. Valencia y Guevara (2013) indican que los polifenoles totales son afectados en su disminución de contenido por el proceso y la temperatura al cual son sometidos, por los métodos de preparación de muestras, por técnica analíticas utilizadas, que pueden influenciar en los resultados obtenidos.

Los antioxidantes son sustancias que tienen propiedades capaces de minimizar los efectos dañinos de los radicales libres. Según Bastias y Cepero (2016), son muy sensibles a la luz, temperatura y oxígeno porque se degradan fácilmente durante el procesamiento $\mathrm{y}$ almacenamiento de los alimentos.
La pérdida de aceptabilidad de un producto por parte del consumidor no necesariamente significa que el producto no sea apto para el consumo, sino que el estándar de calidad establecido ha sido sobrepasado

\section{CONCLUSIONES}

Los compuestos bioactivos de la cáscara de camu camu almacenada durante 45 días a $40{ }^{\circ} \mathrm{C}$ se conservaron mejor con valores de vitamina $\mathrm{C}$ de $56.31 \%$, polifenoles totales $28.21 \%$ y capacidad antioxidante $27.85 \%$.

La energía de activación $(E a)$ requerida para la degradación de los compuestos bioactivos fue de $17.93 \mathrm{Kj} / \mathrm{mol}$ para vitamina $\mathrm{C}, 8.84 \mathrm{Kj} / \mathrm{mol}$ para polifenoles totales y $26.52 \mathrm{Kj} / \mathrm{mol}$ para la capacidad antioxidante.

La vida media útil de vitamina $C$ de la cáscara de camu camu se redujo con el incremento de temperatura de 54 a 35 días. La misma tendencia se observó en polifenoles totales con reducción de 28 a 22 días y para la capacidad antioxidante de 47 a 25 días.

\section{Referencias}

Arellano, E.; Rojas, I.; Paucar, L. 2016. Camu-camu (Myrciaria dubia McVaugh): Fruta tropical de excelentes propiedades funcionales que ayudan a mejorar la calidad de vida. Scientia Agropecuaria. Trujillo 7(4): 433-443. 
Badui, S. 2013. Química de los alimentos. Editor por Marisa de Anta. 5ta ed. Naucalpan de Juárez, México, Pearson educación. 744p.

Bautista, D.; Valdiveiso, M. 2016. Contribucion al estudio de la extraccion de aceites esenciales y secado de cáscara de naranja (Citrus sinensis, var. Valengia Late). Tesis para Ing. De alimentos. Cañete. Perú. Universidad Nacional del Callao. $149 \mathrm{p}$.

Bastías, J.; Cepero, Y. 2016. La vitamina C como un eficaz micronutriente en la fortificación de alimentos. Revista Chilena de nutrición. Chile. 43(1): 81-86.

Busso, C. 2016. Estabilidad de polifenoles y caracterización físico-química y sensorial en pulpas de frutos rojos en relación a los procesos tecnológicos para la obtención de alimentos e ingredientes alimenticios. Dr. Farmacéutico. Buenos Aires. Argentina. Universidad de Buenos Aires Facultad de Farmacia y Bioquimica. 217p.

Cabrera, M. 2015. Cambios en vitamina c, polifenoles totales y capacidad antioxidante en la naranja (Citrus sinensis) durante el almacenamiento. Tesis. Ing. En industrias alimentarias. Tingo María. Perú. Universidad Nacional Agraria de la Selva. $126 \mathrm{p}$.

Caisahuana, M. 2012. Evaluación de vitamina c, polifenoles totales y capacidad antioxidante en dos estados de madurez del camu camu (Myrciaria Dubia Mc Vaugh) de mazamari - satipo. Tesis Ing. En industrias alimentarias. Satipo, Perú, Universidad Nacional del Centro del Perú. 82p.

Castañeda, J.; Arteaga, H.; Siche, R.; Rodirguez, G. 2010 Comparative study of the loss of vitamin $C$ in chalarina (Casimiroa edulis) by four methods of dehydration. Scientia Agropecuaria. Trujillo. 1(1): 75-80.

Cedeño, S. 2017. Efecto de los diferentes procesos de secado en los compuestos bioactivos de murtilla (Ugni molinae Turcz) y su bioaccesibilidad. Tesis de magister. Universidad Austral de Chile. 52p.

Cofré, A. 2015. Determinación de polifenoles totales, actividad antioxidante y antocianinas de jugo de murtilla (Ugni molinae turcz) obtenido por condensación de vapor. Tesis Ing. en alimentos. Valdivia. Chile. Universidad Austral de Chile. $45 \mathrm{p}$.

Cuastumal, H.; Valencia, B.; Ordoñez, L. 2016. Efectos de los tratamientos térmicos en la concentración de vitamina $\mathrm{C}$ y color superficial en tres frutas tropicales. 13(1): 85-93.
Chang, A. 2013. El Camu Camu: Aspectos químicos, farmacológicos y tecnológicos. 1ra ed. Ica, Perú. EISSN. 155p.

Dos-Santos, R.; Cardoso, P.; Vieira, J.; Alves, E.; Pinedo, M.; Bardales, R.; Abanto, C. 2018. Cadeia de produção do camu-camu (Myrciaria dubia Mc Vaugh): o caso das regiões produtoras de loreto e ucayali na Amazônia Peruana. Brazil. 43(4): 261267.

Estrada, H.; Restrepo, C.; Saumett, H.; Perez, L. 2014. Deshidratación osmótica y secado por aire caliente en mango, guayaba y limón para la obtención de ingredientes funcionales. Información tecnológica. 29(3): 197-204.

Fracassetti, D.; Costa, C.; Moulay, L.; Tomás-Barberán, F. 2013. Ellagic acid derivatives, ellagitannins, proanthocyanidins and other phenolic, vitamin C and antioxidant capacity of two powder products from camu-camu fruit (Myrciaria dubia McVaugh). Food Chemistry. 139(1-4): 578-588.

Garay, N.; Villafuerte, E. 2015. Evaluación de diferentes temperaturas de secado en la concentración de antioxidantes y las características organolépticas de la bebida funcional elaborado a partir de la cáscara de camu camu (Myrciaria dubia Mc Vaugh). Tesis Ing. Agroindustrial. Pucallpa, Perú. Universidad Nacional Intercultural De La Amazonía. 108 p.

Gonzales, G.; Pirovani, M.; Piagentini, A.; UlínMontejo, F.; Miranda, E.; Osorio, R.; Maldonado, E.; Salinas, R. 2016. Cinética de cambios sensoriales y vida de anaquel de carambola mínimamente procesada. Rev. Fitotec. Mex. 39(4): $393-402$.

Hung, CH.Y; Yen, G.CH. 2002. Antioxidant activity of phenolic compounds isolated from Mesona procumbens Hemsl, J. Agric. Food chem 50(10): 2993-2997.

Klinar, S.; Chang, A.; Chanllio, J. 2009. Evaluación comparativa de contenido de vitamina $\mathrm{C}$ en diferentes estados de maduración del fruto de camu camu (Myrciaria dubia Mc Vaugh) SEGÚN NTP 011:030:2007. Rev. Cienc. FITOICA. 4(1): 2332.

Mendoza-Corvis, F.; Arteaga-Márquez, M.; PérezSierra, O. 2017. Degradación de la vitamina C en un producto de mango (Mangifera indica L.) y lactosuero. corpoica. ciencia y tecnología agropecuaria. 18(1): 125-137.

Monteiro, U. 2014. Validación del método de determinación del ácido ascórbico y tratamientos con extractos enzimáticos en pulpa de (Myrciaria 
dubia McVaugh) camu. Tesis Ing. Industrias alimentarias. Iquitos. Universidad Nacional de la Amazonia Peruana. 90p.

Muñoz, W.; Chavez, W.; Pabón, C.; Rendón, M.; Patricia-Chaparro, M.; Otálvaro-Álvarez, Á. 2015. Extracción de compuestos fenólicos con actividad antioxidante a partir de Champa (Campomanesia lineatifolia). Rev. Cenic. Ciencias Quím. La Habana. Cuba. 46: 38-46.

Neves, L.; Xavier, V.; Alves, E.; Barcelar, C.; Ruffo, S. 2015. Determining the harvest time of camu-camu (Myrciaria dubia Mc Vaugh) using measured preharvest attributes. Scientia Horticulturae 186: 1523.

Paucar, C. 2012. Caracterización y evaluación antioxidante de la pulpa de camu camu (Myrciaria Dubia Mc vaugh) fresca, concentrada al vacío y almacenada en congelación. Tesis Ing. En industria alimentarias. Tingo María. Perú. Universidad Nacional Agraria de la selva. 101p.

Ramírez, M.; Salgado, N.; Orrego, C. 2012. Conservación de polifenoles en un jugo de fruta modelo secado por Aspersión y liofilización. Rev. Cienc. De las América Latina. 19(1):87-89.

Sánchez, L.; Chávez, J.; Ríos, L.; Cardona, S. 2015. Evaluación de un antioxidante natural extraído del marañón (Anacardium occidentale L.) para mejorar la estabilidad oxidativa del biodiesel de jatropha. Rev. Información tecnológica. 26(6):1930.

Sotero, V.; Silva, L.; García, D.; Imán, S. 2009. Evaluación de la actividad antioxidante de la pulpa, cáscara y semilla del fruto del camu camu (Myrciaria dubia Mc vaugh). Rev. Soc. Quím. Perú Lima. 75(1): 3.

Soto, E.; Barraza, G. 2014. Efecto de la temperatura y tiempo de almacenamiento en las características fisicoquímicas y capacidad antioxidante de pulpa de guayaba (Psidium guajava L.) variedad criolla roja. Rev. Cientifi-k. 2(2): 44-55.

Terry, V. 2015. Deterioro de vitamina c en la pulpa de camu camu (Myrciaria dubia Mc Vaugh) pasteurizada almacenada en funcion del tiempo y la temperatura de almacenamiento. Rev. Investig. Uni. Le Cordon Bleu. 2(2): 39-49.

Torres, Y.; Vidaurre, J. 2015. Cinética de degradación de compuestos fenoles y antiocianinas en una bebida funcional a base de arandano azul (Vaccinium corymbosum L.). Rev. Ingeniería. Ciencia. Tecnología y innovación. 2(2): 7-13.

Valcarcel, J. 2014. Obtencion de los parámetros de secado de la cascara de camu camu (Myrciaria dubia Mc Vaugh) en un lecho fluidizado. Tesis. Ing en industrias alimentarias. Iquitos. Perú. Universidad Nacional de la Amazonia Peruana. $105 p$.

Valdés-Hernández, G.; Cruz-Viera, L.; CometRodríguez, R. 2015. Influencia de las condiciones de operación en la extracción de polifenoles a partir de hojas de Moringa oleifera Lam. Revista CENIC. Ciencias Químicas. 46(1):135-145.

Valencia, C.; Guevara, A. 2013. Variación de la capacidad antioxidante y compuestos bioactivos durante el procesamiento del néctar de zarzamora (Rubus fructicosus L.). Revista de la Sociedad Química del Perú 79 (2): 116-125.

Villareal, Y.; Fernado, D.; Osorio, O.; Felipe, A. 2013. Efecto de pasteurización sobre características sensoriales y contenido de vitamina $C$ en jugos de frutas. 11(2); 66-75.

Zapata, K.; Rojano, B.; Cortes, F. 2015. Efecto térmico del secado por aspersión sobre los metabolitos antioxidantes de la curuba larga (Passiflora mollisima baley). 26(1): 77-84. 